- State of -The-Art

\title{
Bringing-Up A Free Mind in Education
}

\section{Raouf Sallam}

A surgeon interested and involved in education and training, commented positively on one of my previous editorials in which I said that '.... the wrong content will very easily cross the line from education to brain wash'. He hence asked,' how to know that I am not brain washing people while claiming educating them??'

I rephrased his question into: 'how to avoid brain wash during education??' To this I would say: -

The best protection against brain wash in general is a free mind developed during education. While noting a balanced, peer reviewed, unbiased, unregimented and malleable content is essential, remember that the process is equally important. For adult education I have the following suggestions regarding the process:

1- Make your education learning based rather than teaching based. A student looking-up for information himself- or in a study group- will pick-up a personalized bouquet of information that teaching may then help arrange and transform to knowledge.

2- Base your teaching on facts and established knowledge as much as you can.

3- When using sub-factual knowledge- and you will inevitably have to- draw the attention of the students to the degree of validity of the information used.

4- Tell the students how the information at hand was arrived to.

For example, when you tell the student that the composition of human breast milk is [protein...., fat..... etc.] he may consider this as the definite and final list of the substances in the milk. But when you tell him that we arrived to this list by testing for the substances we know and know how to test for, he will realize that there might be other substances in the human breast milk that awaits discovery. 
Another example, when you tell the student about the normal range of e.g. blood urea or blood sugar in the healthy adult, he will consider any figure outside the range as abnormal and hence pathological. But if you tell him that most of these ' normal ranges' are in fact a computation of the mean and standard deviation of the readings in normal subjects he will realize that $5 \%$ of the normal subjects will have readings outside the ' normal range'.

5- Ask before you tell. For example, after discussing the facts about acute appendicitis ask them what they think the treatment should be. You may get some interesting answers; one of these may be the treatment of acute appendicitis in the future.

6- Provoke, challenge and even instruct your students to find alternative answers or solutions for the problem at hand, even if these solutions seem less satisfactory than the current accepted one.

7- Challenge them with the question ' what if the standard information or solution is not available or not applicable?'

8- While teaching liberally and appropriately use the following humbling phrases: I do not know, I am not sure, this is what I know, and I will look it up and tell you later.

9- Remind your students that knowledge is ours to use, augment and modify; analyses and utilize and that we may yield to established knowledge, but we should not submit to it.

10- Remind them- with examples- that the best does not remain the best for long and that there is always a' better ' in the horizon.

11- Remind them that Newton's theory was incomplete, and that Einstein was wrong at least once and Freud was wrong many times and Darwin will probably prove wrong all the way through. Remind them that Galileo and Copernicus were absolutely right when they said that the earth moves and is not the center of the universe but at the same time they were equally wrong when they said that the sun does not move and is the center of the universe.

Good luck.

Prof. Raouf Sallam F.R.C.S. 